\title{
Retrieval of Shape using Curves and Contours
}

\author{
Dariusz Jacek Jakóbczak*
}

\author{
Department of Electronics and Computer Science, Koszalin University of Technology \\ Sniadeckich 2, 75-453 Koszalin, Poland \\ E-mail: dariusz.jakobczak@tu.koszalin.pl
}

\begin{abstract}
Proposed method is dealing with multi-dimensional data modeling, extrapolation and interpolation using the set of high-dimensional feature vectors. Identification of handwriting, signature, faces or fingerprints need data modeling and each model of the pattern is built by a choice of characteristic key points and multi-dimensional modeling functions. Novel modeling via nodes combination and parameter $\gamma$ as $N$ dimensional function enables data parameterization and interpolation for feature vectors. Multidimensional data is modeled and interpolated via different functions for each feature: polynomial, sine, cosine, tangent, cotangent, logarithm, exponent, arc sin, arc cos, arc tan, arc cot or power function.
\end{abstract}

Keywords: image retrieval; pattern recognition; data modeling; vector interpolation; PFC method; feature reconstruction; probabilistic modeling

\section{INTRODUCTION}

The idea of paper is connected with different curve modeling for the same set of curve points (nodes). The problem of multidimensional data modeling appears in many branches of science and industry. Image retrieval, data reconstruction; object identification or pattern recognition are still the open problems in artificial intelligence and computer vision. The paper is dealing with these questions via modeling of high-dimensional data for applications of image segmentation in image retrieval and recognition tasks. Handwriting based author recognition offers a huge number of significant implementations which make it an important research area in pattern recognition. There are so many possibilities and applications of the recognition algorithms that implemented methods have to be concerned on a single problem: retrieval, identification, verification or recognition. This paper is concerned with two parts: image retrieval and recognition tasks. Image retrieval is based on modeling of unknown features via combination of $\mathrm{N}$-dimensional functions for each feature. In the case of biometric writer recognition, each person is represented by the set of modeled letters or symbols. The sketch of proposed method consists of three steps: first handwritten letter or symbol must be modeled by a vector of features ( $N$-dimensional data), then compared with unknown letter and finally there is a decision of identification. Author recognition of handwriting and signature is based on the choice of feature vectors and modeling functions. So high-dimensional data interpolation in handwriting identification [20] is not only a pure mathematical problem but important task in pattern recognition and artificial intelligence such as: biometric recognition, personalized handwriting recognition [3-5], automatic forensic document examination [6,7], classification of ancient manuscripts [8]. Also writer recognition [9] in monolingual handwritten texts is an extensive area of study and the methods independent from the language are well-seen [10-13]. Proposed method represents languageindependent and text-independent approach because it identifies the author via a set of letters or symbols from the sample.
Writer recognition methods in the recent years are going to various directions [14-18]: writer recognition using multi-script handwritten texts, introduction of new features, combining different types of features, studying the sensitivity of character size on writer identification, investigating writer identification in multi-script environments, impact of ruling lines on writer identification, model perturbed handwriting, methods based on run-length features, the edge-direction and edge-hinge features, a combination of codebook and visual features extracted from chain code and polygonized representation of contours, the autoregressive coefficients, codebook and efficient code extraction methods, texture analysis with Gabor filters and extracting features, using Hidden Markov Model [19] or Gaussian Mixture Model [1]. So hybrid soft computing is essential: no method is dealing with writer identification via $\mathrm{N}$-dimensional data modeling or interpolation and multidimensional points comparing as it is presented in this paper. The paper wants to approach a problem of curve interpolation and shape modeling by characteristic points in handwriting identification [2]. Proposed method relies on nodes combination and functional modeling of curve points situated between the basic set of key points. The functions that are used in calculations represent whole family of elementary functions with inverse functions: polynomials, trigonometric, cyclometric, logarithmic, exponential and power function. Nowadays methods apply mainly polynomial functions, for example Bernstein polynomials in Bezier curves, splines [25] and NURBS. But Bezier curves don't represent the interpolation method and cannot be used for example in signature and handwriting modeling with characteristic points (nodes). Numerical methods [21-23] for data interpolation are based on polynomial or trigonometric functions, for example Lagrange, Newton, Aitken and Hermite methods. These methods have some weak sides and are not sufficient for curve interpolation in the situations when the curve cannot be built by polynomials or trigonometric functions [24]. 
This paper presents novel method of high-dimensional interpolation in hybrid soft computing and takes up method of multidimensional data modeling. The method requires information about data (image, object, and curve) as the set of $\mathrm{N}$-dimensional feature vectors. So this paper wants to answer the question: how to retrieve the image using $\mathrm{N}$-dimensional feature vectors and to recognize a handwritten letter or symbol by a set of highdimensional nodes via hybrid soft computing?

\section{MULTIDIMENSIONAL MODELING OF FEATURE VECTORS}

Proposed method is computing (interpolating) unknown (unclear, noised or destroyed) values of features between two successive nodes ( $\mathrm{N}$-dimensional vectors of features) using hybridization of mathematical analysis and numerical methods, Calculated values (unknown or noised features such as coordinates, colors, textures or any coefficients of pixels, voxels and doxels or image parameters) are interpolated and parameterized for real number $\alpha_{i} \in[0 ; 1](i=1,2, \ldots N-1)$ between two successive values of feature. This method uses the combinations of nodes $\left(N\right.$-dimensional feature vectors) $p_{1}=\left(x_{1}, y_{1}, \ldots, Z_{1}\right)$, $p_{2}=\left(x_{2}, y_{2}, \ldots, Z_{2}\right), \ldots, \quad p_{n}=\left(x_{n}, y_{n}, \ldots z_{n}\right) \quad$ as $h\left(p_{1}, p_{2}, \ldots, p_{m}\right) \quad$ and $m=1,2, \ldots n$ to interpolate unknown value of feature (for example $y$ ) for the rest of coordinates:

$c_{1}=\alpha_{1} \cdot x_{k}+\left(1-\alpha_{1}\right) \cdot x_{k+1}, \ldots \ldots c_{N-1}=\alpha_{N-1} \cdot Z_{k}+\left(1-\alpha_{N-1}\right) \cdot z_{k+1}$, $k=1,2, \ldots n-1$,

$c=\left(c_{1}, \ldots, c_{N-1}\right), \quad \alpha=\left(\alpha_{1}, \ldots, \alpha_{N-1}\right), \quad \gamma_{i}=F_{i}\left(\alpha_{i}\right) \in[0 ; 1]$, $i=1,2, \ldots N-1$

$y(c)=\gamma \cdot y_{k}+(1-\gamma) y_{k+1}+\gamma(1-\gamma) \cdot h\left(p_{1}, p_{2}, \ldots, p_{m}\right)$

$\alpha_{i} \in[0 ; 1], \gamma=F(\alpha)=F\left(\alpha_{1}, \ldots, \alpha_{N-1}\right) \in[0 ; 1]$.

Then $N-1$ features $c 1, \ldots, c_{N-1}$ are parameterized by $\alpha_{1}, \ldots$, $\alpha_{N-1}$ between two nodes and the last feature (for example $y$ ) is interpolated via formula (1). Of course there can be calculated $x(c)$ or $z(c)$ using (1). Two examples of $h$ (when $N=2$ ) computed for MHR method [26] with good features because of orthogonal rows and columns at Hurwitz-Radon family of matrices:

$$
h\left(p_{1}, p_{2}\right)=\frac{y_{1}}{x_{1}} x_{2}+\frac{y_{2}}{x_{2}} x_{1}
$$

or

$$
\begin{aligned}
& h\left(p_{1}, p_{2}, p_{3}, p_{4}\right)=\frac{1}{x_{1}^{2}+x_{3}^{2}}\left(x_{1} x_{2} y_{1}+x_{2} x_{3} y_{3}+x_{3} x_{4} y_{1}-x_{1} x_{4} y_{3}\right)+ \\
& +\frac{1}{x_{2}^{2}+x_{4}^{2}}\left(x_{1} x_{2} y_{2}+x_{1} x_{4} y_{4}+x_{3} x_{4} y_{2}-x_{2} x_{3} y_{4}\right)^{.}
\end{aligned}
$$

The simplest nodes combination is

$$
h\left(p_{1}, p_{2}, \ldots, p_{m}\right)=0
$$

and then there is a formula of interpolation:

$$
y(c)=\gamma \cdot y_{i}+(1-\gamma) y_{i+1} .
$$

Formula (1) gives the infinite number of calculations for unknown feature determined by choice of $F$ and $h$. Nodes combination is the individual feature of each modeled data. Coefficient $\gamma=F(\alpha)$ and nodes combination $h$ are key factors in data interpolation and object modeling.

\section{$\mathrm{N}$-dimensional functions in modeling}

Unknown values of features, settled between the nodes, are computed using (1). Key question is dealing with coefficient $\gamma$. The simplest way of calculation means $h=$ 0 and $\gamma_{i}=\alpha_{i}$. Then proposed method represents a linear interpolation. Each interpolation requires specific values of $\alpha_{i}$ and $\gamma$ in (1) depends on parameters $\alpha_{i} \in$ $[0 ; 1]$ :

$\gamma=F(\alpha), F:[0 ; 1]^{N-1} \rightarrow[0 ; 1], F(0, \ldots, 0)=0, F(1, \ldots, 1)=1$

and $F$ is strictly monotonic for each $\alpha_{i}$ separately. Coefficient $\gamma_{i}$ are calculated using appropriate function and choice of function is connected with initial requirements and data specifications. Different values of coefficients $\gamma_{i}$ are connected with applied functions $F_{i}\left(\alpha_{i}\right)$. These functions $\gamma_{i}=F_{i}\left(\alpha_{i}\right)$ represent the examples of modeling functions for $\alpha_{i} \in[0 ; 1]$ and real number $s>$ $0, i=1,2, \ldots N-1$. Each function is applied for different modelling:

$\gamma_{i}=\alpha_{i}^{s}, \gamma_{i}=\sin \left(\alpha_{i}^{s} \cdot \pi / 2\right), \gamma_{i}=\sin ^{s}\left(\alpha_{i} \cdot \pi / 2\right), \gamma_{i}=1-\cos \left(\alpha_{i}^{s} \cdot \pi / 2\right)$, $\gamma_{i}=1-\cos ^{s}\left(\alpha_{i} \cdot \pi / 2\right), \gamma_{i}=\tan \left(\alpha_{i}^{s} \cdot \pi / 4\right), \gamma_{i}=\tan ^{s}\left(\alpha_{i} \cdot \pi / 4\right)$,

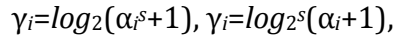
$\gamma_{i}=\left(2^{\alpha}-1\right)^{s}, \gamma_{i}=2 / \pi \cdot \arcsin \left(\alpha_{i}^{s}\right), \gamma_{i}=\left(2 / \pi \cdot \arcsin \alpha_{i}\right)^{s}$, $\gamma_{i}=1-2 / \pi \cdot \arccos \left(\alpha_{i}^{s}\right), \quad \gamma_{i}=1-\left(2 / \pi \cdot \arccos \alpha_{i}\right)^{s}$, $\gamma_{i}=4 / \pi \cdot \arctan \left(\alpha_{i}{ }^{s}\right), \quad \gamma_{i}=\left(4 / \pi \cdot \arctan \alpha_{i}\right)$, $\gamma_{i}=\left(\pi / 2-\alpha_{i} \cdot \pi / 4\right), \quad \gamma_{i}=\operatorname{ctg}^{s}\left(\pi / 2-\alpha_{i} \cdot \pi / 4\right)$, $\gamma_{i}=2-4 / \pi \cdot \operatorname{arcctg}\left(\alpha_{i}^{s}\right), \gamma_{i}=\left(2-4 / \pi \cdot \operatorname{arcctg} \alpha_{i}\right)^{s}$

or any strictly monotonic function between points $(0 ; 0)$ and $(1 ; 1)$. For example interpolations of function $y=2^{x}$ for $N=2, h=0$ and $\gamma=\alpha^{s}$ with $s=0.8 \mathrm{ctg}$ (FIGURE 1) is much better than linear interpolation.

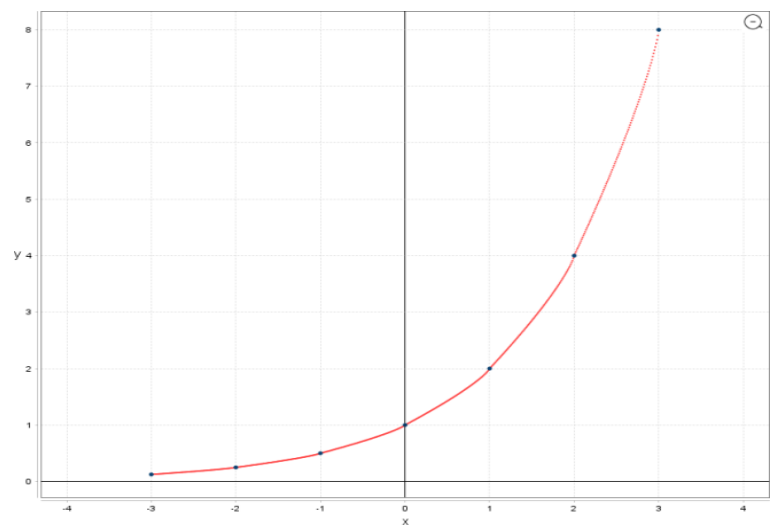

FIGURE 1: Two-dimensional modeling of function $y=2^{x}$ with seven nodes and $h=0, \gamma=\alpha^{0.8}$

Functions $\gamma_{i}$ are strictly monotonic for each variable $\alpha_{i}$ $\in[0 ; 1]$ as $\gamma=F(\alpha)$ is $N$-dimensional modeling function, for example:

$$
\gamma=\frac{1}{N-1} \sum_{i=1}^{N-1} \gamma_{i}, \quad \gamma=\prod_{i=1}^{N-1} \gamma_{i}
$$

and every monotonic combination of $\gamma_{i}$ such as

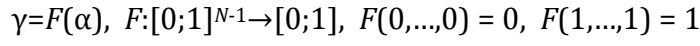

For example when $N=3$ there is a bilinear interpolation:

$$
\gamma_{1}=\alpha_{1}, \gamma_{2}=\alpha_{2}, \gamma=1 / 2\left(\alpha_{1}+\alpha_{2}\right)
$$

or a bi-quadratic interpolation:

$$
\gamma_{1}=\alpha_{1}^{2}, \gamma_{2}=\alpha_{2}^{2}, \gamma=1 / 2\left(\alpha_{1}^{2}+\alpha_{2}^{2}\right)
$$


or a bi-cubic interpolation:

$$
\gamma_{1}=\alpha_{1}{ }^{3}, \gamma_{2}=\alpha_{2}{ }^{3}, \gamma=1 / 2\left(\alpha_{1}{ }^{3}+\alpha_{2}{ }^{3}\right)
$$

or others modeling functions $\gamma$. Choice of functions $\gamma_{i}$ and value $s$ depends on the specifications of feature vectors and individual requirements. What is very important: two data sets (for example a handwritten letter or signature) may have the same set of nodes (feature vectors: pixel coordinates, pressure, speed, angles) but different $h$ or $\gamma$ results in different interpolations (Fig.2-4). Here are three examples of reconstruction (Fig.2-4) for $N=2$ and four nodes: $(-1.5 ;-1),(1.25 ; 3.15),(4.4 ; 6.8)$ and $(8 ; 7)$.

Formula of the curve is not given. Algorithm of proposed retrieval, interpolation and modeling consists of five steps: first choice of nodes $p_{i}$ (feature vectors), then choice of nodes combination $h\left(p_{1}, p_{2}, \ldots, p_{m}\right)$, choice of modeling function $\gamma=F(\alpha)$, determining values of $\alpha_{i} \in[0 ; 1]$ and finally the computations (1)

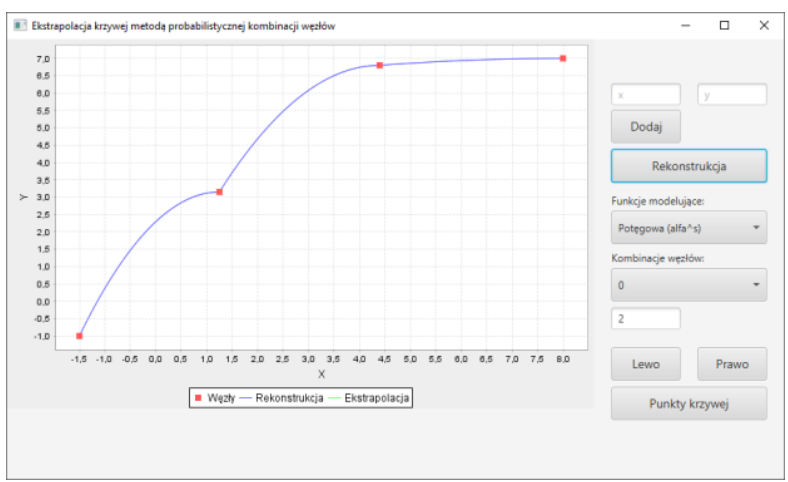

FIGURE 2: 2D modeling for $\gamma=\alpha^{2}$ and $h=0$

And other interpolations for the same set of nodes:

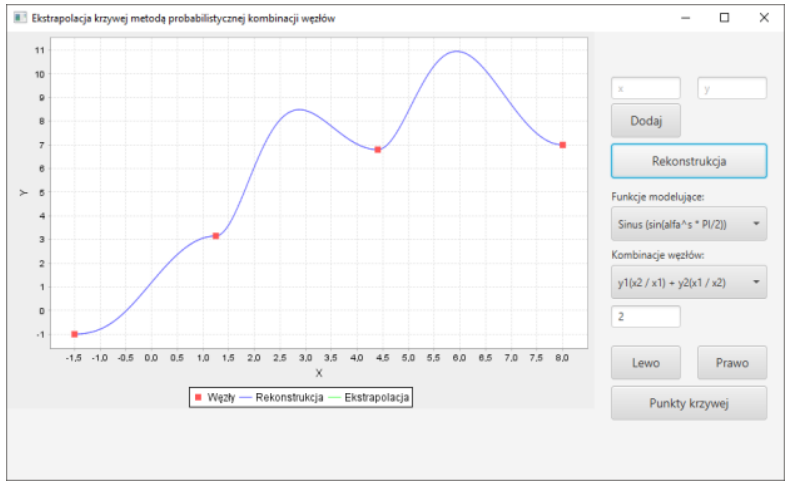

FIGURE 3: 2D reconstruction for $\gamma=\sin \left(\alpha^{2} \cdot \pi / 2\right)$ and $h$ in (2)

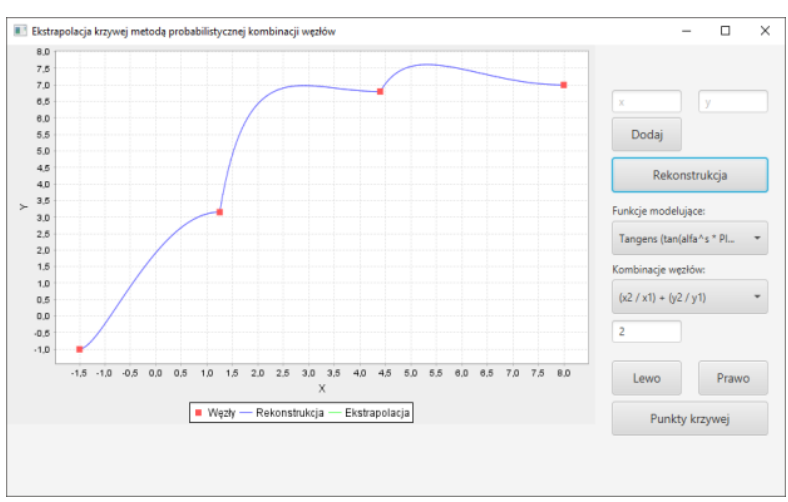

FIGURE 4: $2 D$ interpolation for $\gamma=\tan \left(\alpha^{2} \cdot \pi / 4\right)$ and $h=\left(x_{2} / x_{1}\right)+\left(y_{2} / y_{1}\right)$
So there are different data reconstructions with different modeling functions. As it can be observed, there is one extremum between two nodes for modeling with $\mathrm{h} \neq 0$ (Fig.3-4). Comparing with polynomial or spline interpolations, there is one very important question: how to avoid extremum between each pair of nodes and how to minimize interpolation error? Generally current methods do not answer this key question. Nowadays methods of interpolations rely mainly on polynomials, especially on cubic splines. It means that there are interpolation polynomials $W(x)$ of degree 3 for every range of two successive interpolation nodes $\left(x_{\mathrm{i}}, y_{\mathrm{i}}\right)$ and $\left(x_{i+1}, y_{i+1}\right)$. This method of cubic splines is $C^{2}$ class this fact is very important in many applications of cubic interpolation. But second important feature of this method is interpolation error for function $f(x)$ :

$$
\begin{aligned}
& |f(x)-W(x)| \leq 5 M\left|\left(x-x_{i}\right)\left(x-x_{i+1}\right)\right|, \\
& M=\sup _{x \in[a, b]}\left|f^{\prime \prime}(x)\right| .
\end{aligned}
$$

So interpolation error depends on second derivative in the range of nodes $[a ; b]$ and this value cannot be estimated in general. Cubic spline can have extremum and may differ from interpolated function $f(x)$ very much. Also interpolation polynomial $W_{n}(x)$ of degree $n$ (Lagrange or Newton) for $n+1$ nodes $\left(x_{0}, y_{0}\right),\left(x_{1}, y_{1}\right) \ldots$ $\left(x_{n}, y_{n}\right)$ is connected with unpredictable error in general with calculations of derivative rank $n+1$ :

$$
\begin{aligned}
& \left|f(x)-W_{n}(x)\right| \leq \frac{M_{n+1}}{(n+1) !}\left|\left(x-x_{o}\right)\left(x-x_{1}\right) \ldots\left(x-x_{n}\right)\right|, \\
& M_{n+1}=\sup _{x \in[a, b]}\left|f^{(n+1)}(x)\right| .
\end{aligned}
$$

Proposed method with $h=0$ and $\alpha \in[0 ; 1]$ represents formulas as convex combinations of nodes' coordinates:

$$
x(\alpha)=\alpha \cdot x_{k}+(1-\alpha) x_{k+1}, y(\alpha)=\gamma_{k} \cdot y_{k}+\left(1-\gamma_{k}\right) y_{k+1} .
$$

and interpolation error in general between two nodes looks as follows:

$$
\varepsilon_{k} \leq\left|y_{k+1}-y_{k}\right|
$$

Proposed method is dealing with such significant features:

- no extremum between two nodes;

- interpolation error does not depend on the value of derivative in the nodes or outside the nodes (even if derivative does not exist);

- interpolated function can be smooth in the nodes (class $\mathrm{C}^{1}$ );

- reconstruction of the function that much differs from the shape of polynomial, and not only function but any curve, also closed;

- extrapolation is calculated with the same formulas for $\alpha \notin[0 ; 1]$

- the idea of linear interpolation is applied for other modeling functions, not only $\gamma=\alpha^{1}$;

- convexity between the nodes is fixed using two modeling functions:

$$
\begin{gathered}
\gamma_{k}=\alpha^{s} \text { or } \gamma_{k}=\sin \left(\alpha^{s \cdot \pi} / 2\right) \\
\text { with real parameter } s>0
\end{gathered}
$$

These two kinds of modeling functions are the simplest function, chosen via many calculations as follows:

- $\gamma_{k}=\alpha^{s}$ if convexity is not changing between the nodes $\left(x_{\mathrm{k}}, y_{\mathrm{k}}\right)$ and $\left(x_{k+1}, y_{k+1}\right)$

- $\gamma_{k}=\sin \left(\alpha^{s \cdot \pi} \cdot \pi\right)$ if convexity is changing between the nodes $\left(x_{\mathrm{k}}, y_{\mathrm{k}}\right)$ and $\left(x_{k+1}, y_{k+1}\right)$ 


\section{THEOREM If:}

1. There are given nodes of continuous function $y=f(x):\left(x_{0}, y_{0}\right),\left(x_{1}, y_{1}\right) \ldots\left(x_{n}, y_{\mathrm{n}}\right), n \geq 2$;

2. There are formulas to calculate values between the nodes:

$$
\begin{aligned}
& x(\alpha)=\alpha \cdot x_{k}+(1-\alpha) x_{k+1}, \\
& y(\alpha)=\gamma_{k} \cdot y_{k}+\left(1-\gamma_{k}\right) y_{k+1} .
\end{aligned}
$$

$\alpha \in[0 ; 1], k=2,3 \ldots n-1, \gamma_{k}=\alpha^{s}$ or $\gamma_{k}=\sin \left(\alpha^{s} \cdot \pi / 2\right)$ with real parameter $s>0$;

3. Three successive nodes are monotonic, for example let's assume:

$$
y_{0}>y_{1}>y_{2} \text { or } y_{0}<y_{1}<y_{2}
$$

Then there is the method of 2D curve interpolation and extrapolation such as:

T.1: There is no extremum between two successive nodes - interpolated function is monotonic in the range of two nodes.

T.2: Interpolated curve is class $\mathrm{C}^{0}$ (continuous) or $\mathrm{C}^{1}$ (continuous and smooth).

T.3: Interpolation error does not depend on the value of derivative in the nodes or outside the nodes (even if derivative does not exist).

T.4: Convexity between two nodes $\left(x_{\mathrm{k}}, y_{\mathrm{k}}\right)$ and $\left(x_{\mathrm{k}+1}, y_{\mathrm{k}+1}\right)$ is fixed using modeling functions $\gamma_{k}=\alpha^{s}$ (if convexity is not changing) or $\gamma_{k}=\sin \left(\alpha^{s \cdot \pi / 2)}\right.$ (if convexity is changing).

T.5: Extrapolation is calculated with the same formulas for $\alpha \notin[0 ; 1]$.

\section{Proof:}

T.1: Convex combination to calculate $x(\alpha)$ and $y(\alpha)$ between two nodes with strictly monotonic function $\gamma_{k}$ gives us monotonic interpolation of the curve with no extremum between two nodes.

T.2: Interpolated curve is class $\mathrm{C}^{0}$ (continuous) just from definition of $x(\alpha)$ and $y(\alpha)$. Also smooth interpolation between nodes is achieved with the same. Only smooth function in the inner nodes must be proved. Here is shown how to achieve smooth function in the inner nodes - let's assume then $y_{\mathrm{k}} \neq y_{\mathrm{k}+1}$ for each $k$. If $y_{\mathrm{k}}=y_{\mathrm{k}+1}$ for any $k$, then according to T.1 there must be the simplest linear interpolation between nodes $\left(x_{\mathrm{k}}, y_{\mathrm{k}}\right)$ and $\left(x_{\mathrm{k}+1}, y_{\mathrm{k}+1}\right)$ and interpolated curve is not smooth in nodes $\left(x_{\mathrm{k}}, y_{\mathrm{k}}\right)$ and $\left(x_{\mathrm{k}+1}, y_{\mathrm{k}+1}\right)$

For first three monotonic nodes $\left(x_{0}, y_{0}\right),\left(x_{1}, y_{1}\right)$ and $\left(x_{2}, y_{2}\right)$ there are calculations to fix parameter $\mathrm{s}$ for modeling function $\gamma_{1}$ between nodes $\left(x_{0}, y_{0}\right)$ and $\left(x_{2}, y_{2}\right)$ interpolating node $\left(x_{1}, y_{1}\right)$ inside:

$$
\alpha=\frac{x_{2}-x_{1}}{x_{2}-x_{0}} \in(0 ; 1), \quad t=\frac{y_{2}-y_{1}}{y_{2}-y_{0}} \in(0 ; 1) \text {. }
$$

If convexity is not changing between $\left(x_{0}, y_{0}\right)$ and $\left(x_{2}, y_{2}\right)$, then $\gamma_{1}=\alpha^{s}$ and $s=\log _{\alpha} t$.

If convexity is changing between $\left(x_{0}, y_{0}\right)$ and $\left(x_{2}, y_{2}\right)$, then $\gamma_{1}=\sin \left(\alpha^{s \cdot \pi / 2)}\right.$ and $s=\log _{\alpha}\left(\frac{2}{\pi} \arcsin t\right)$.

A1 (beginning of the loop in algorithm for $k=2,3 \ldots n-1$ ): Having modeling function $\gamma_{1}$ between nodes $\left(x_{0}, y_{0}\right)$ and $\left(x_{2}, y_{2}\right)$, it is possible for any $\alpha^{*} \rightarrow 0$ calculate

$x\left(\alpha^{*}\right)=\alpha^{*} \cdot x_{0}+\left(1-\alpha^{*}\right) x_{2}, y\left(\alpha^{*}\right)=\gamma_{1} \cdot y_{0}+\left(1-\gamma_{1}\right) y_{2}$.
Then left difference quotient $c$ is computed in the node $\left(x_{2}, y_{2}\right)$ :

$$
c=\frac{y_{2}-y\left(\alpha^{*}\right)}{x_{2}-x\left(\alpha^{*}\right)} .
$$

Of course if value of derivative in $\left(x_{2}, y_{2}\right)$ is known, $c=f^{\prime}\left(x_{2}\right) \neq 0$. Then parameter $u$ is fixed to obtain left $(c)$ and right difference quotient equal in $\left(x_{2}, y_{2}\right)$ - it means smooth in this node. If $y_{3}$ preserves the same monotonicity like $y_{2}$ and $y_{1}\left(y_{1}>y_{2}>y_{3}\right.$ or $\left.y_{1}<y_{2}<y_{3}\right)$ then

$$
u=1-c\left(1-\alpha^{*}\right) \frac{x_{3}-x_{2}}{y_{3}-y_{2}} .
$$

If $y_{3}$ does not preserve the same monotonicity like $y_{2}$ and $y_{1}$ then (because of different sign of left and right difference quotient)

$$
u=1+c\left(1-\alpha^{*}\right) \frac{x_{3}-x_{2}}{y_{3}-y_{2}} .
$$

And as it was: if convexity is not changing between $\left(x_{2}, y_{2}\right)$ and $\left(x_{3}, y_{3}\right)$, then $\gamma_{2}=\alpha^{s}$ and

$$
s=\log _{\alpha^{*}} u .
$$

If convexity is changing between $\left(x_{2}, y_{2}\right)$ and $\left(x_{3}, y_{3}\right)$, then $\gamma_{2}=\sin \left(\alpha^{s \cdot \pi} / 2\right)$ and

$$
s=\log _{\alpha^{*}}\left(\frac{2}{\pi} \arcsin u\right) .
$$

So smooth interpolation function in the node $\left(x_{2}, y_{2}\right)$ is achieved. And smooth interpolation for next range of nodes $\left(x_{3}, y_{3}\right)$ and $\left(x_{4}, y_{4}\right)$ is starting like loop $\mathbf{A 1}$ for $k=3$. And so on till last range of nodes $\left(x_{n-1}, y_{n-1}\right)$ and $\left(x_{n}, y_{n}\right)$ for $k=n-1$ in $\mathbf{A 1}$.

T.3: According to T.1 - interpolation error between two nodes for each $k$ is equal:

$$
\varepsilon_{k} \leq\left|y_{k+1}-y_{k}\right| \text {. }
$$

T.4: These modeling functions are the simplest functions to achieve convexity changing or not.

T.5: Extrapolation left of first node $\left(x_{0}, y_{0}\right)$ is done with modeling function $\gamma_{1}$ and $\alpha>1$. Extrapolation right of last node $\left(x_{n}, y_{\mathrm{n}}\right)$ is done with modeling function $\gamma_{n-1}$ and $\alpha<0$. Then modeling function $\gamma_{n-1}$ must have domain with $\alpha<0$. If not, there is possibility to define:

$$
\begin{aligned}
& x(\alpha)=\alpha \cdot x_{k+1}+(1-\alpha) x_{k}, \\
& y(\alpha)=\gamma_{k} \cdot y_{k+1}+\left(1-\gamma_{k}\right) y_{k} .
\end{aligned}
$$

This theorem describes main features of proposed method.

\section{CONCLUSIONS}

The autor's method enables interpolation and modeling of high-dimensional data using features' combinations and different coefficients $\gamma$ : polynomial, sinusoidal, cosinusoidal, tangent, cotangent, logarithmic, exponential, arc sin, arc cos, arc tan, arc cot or power function. Functions for $\gamma$ calculations are chosen individually at each data modeling and it is treated as $\mathrm{N}$-dimensional function: $\gamma$ depends on initial requirements and features' specifications. Novel method leads to data interpolation as handwriting or signature identification and image retrieval via discrete set of feature vectors in $N$-dimensional feature space. So this method makes 
possible the combination of two important problems: interpolation and modeling in a matter of image retrieval or writer identification. Main features of the method are: this interpolation develops a linear interpolation in multidimensional feature spaces into other functions as $\mathrm{N}$-dimensional functions; nodes combination and coefficients $\gamma$ are crucial in the process of data parameterization and interpolation: they are computed individually for a single feature; modeling of closed curves.

\section{REFERENCES}

[1] Schlapbach, A., Bunke, H.: Off-line writer identification using Gaussian mixture models. In: International Conference on Pattern Recognition, pp. 992-995 (2006)

[2] Bulacu, M., Schomaker, L.: Text-independent writer identification and verification using textural and allographic features. IEEE Trans. Pattern Anal. Mach. Intell. 29 (4), 701-717 (2007)

[3] Djeddi, C., Souici-Meslati, L.: A texture based approach for Arabic writer identification and verification. In: International Conference on Machine and Web Intelligence, pp. 115-120 (2010)

[4] Djeddi, C., Souici-Meslati, L.: Artificial immune recognition system for Arabic writer identification. In: International Symposium on Innovation in Information and Communication Technology, pp. 159-165 (2011)

[5] Nosary, A., Heutte, L., Paquet, T.: Unsupervised writer adaption applied to handwritten text recognition. Pattern Recogn. Lett. 37 (2), 385-388 (2004)

[6] Van, E.M., Vuurpijl, L., Franke, K., Schomaker, L.: The WANDA measurement tool for forensic document examination. J. Forensic Doc. Exam. 16, 103-118 (2005)

[7] Schomaker, L., Franke, K., Bulacu, M.: Using codebooks of fragmented connected-component contours in forensic and historic writer identification. Pattern Recogn. Lett. 28 (6), 719727 (2007)

[8] Siddiqi, I., Cloppet, F., Vincent, N.: Contour based features for the classification of ancient manuscripts. In: Conference of the International Graphonomics Society, pp. 226-229 (2009)

[9] Garain, U., Paquet, T.: Off-line multi-script writer identification using AR coefficients. In: International Conference on Document Analysis and Recognition, pp. 991-995 (2009)

[10] Bulacu, M., Schomaker, L., Brink, A.: Textindependent writer identification and verification on off-line Arabic handwriting. In: International Conference on Document Analysis and Recognition, pp. 769-773 (2007)

[11] Ozaki, M., Adachi, Y., Ishii, N.: Examination of effects of character size on accuracy of writer recognition by new local arc method. In: International Conference on Knowledge-Based Intelligent Information and Engineering Systems, pp.11701175 (2006)

[12] Chen, J., Lopresti, D., Kavallieratou, E.: The impact of ruling lines on writer identification In: International Conference on Frontiers in Handwriting Recognition, pp. 439-444 (2010)

[13] Chen, J., Cheng, W., Lopresti, D.: Using perturbed handwriting to support writer identification in the presence of severe data constraints. In: Document Recognition and Retrieval, pp. 1-10 (2011)

[14] Galloway, M.M.: Texture analysis using gray level run lengths. Comput. Graphics Image Process. 4 (2), 172-179 (1975)
[15] Siddiqi, I., Vincent, N.: Text independent writer recognition using redundant writing patterns with contour-based orientation and curvature features. Pattern Recogn. Lett. 43 (11), 3853-3865 (2010)

[16] Ghiasi, G., Safabakhsh, R.: Offline text-independent writer identification using codebook and efficient code extraction methods. Image and Vision Computing 31, 379-391 (2013)

[17] Shahabinejad, F., Rahmati, M.: A new method for writer identification and verification based on Farsi/Arabic handwritten texts, Ninth International Conference on Document Analysis and Recognition (ICDAR 2007), pp. 829-833 (2007)

[18] Schlapbach, A., Bunke, H.: A writer identification and verification system using HMM based recognizers, Pattern Anal. Appl. 10, 33-43 (2007)

[19] Schlapbach, A., Bunke, H.: Using HMM based recognizers for writer identification and verification, 9th Int. Workshop on Frontiers in Handwriting Recognition, pp. 167-172 (2004)

[20] Marti, U.-V., Bunke, H.: The IAM-database: an English sentence database for offline handwriting recognition, Int. J. Doc. Anal. Recognit. 5, 39-46 (2002)

[21] Collins II, G.W.: Fundamental Numerical Methods and Data Analysis. Case Western Reserve University (2003)

[22] Chapra, S.C.: Applied Numerical Methods. McGrawHill (2012)

[23] Ralston, A., Rabinowitz, P.: A First Course in Numerical Analysis - Second Edition. Dover Publications, New York (2001)

[24] Zhang, D., Lu, G.: Review of Shape Representation and Description Techniques. Pattern Recognition 1(37), 1-19 (2004)

[25] Schumaker, L.L.: Spline Functions: Basic Theory. Cambridge Mathematical Library (2007)

[26] Jakóbczak, D.J.: 2D Curve Modeling via the Method of Probabilistic Nodes Combination - Shape 